\title{
Evaluation of various techniques for the detection of Nocardia species in clinically evident cases of pulmonary and extra- pulmonary tuberculosis
}

\author{
Rajesh Bareja ${ }^{1}$, Ramesh Chand Jindal ${ }^{2}$, Prakriti Vohra ${ }^{3}$, Prem Singh Grover ${ }^{4}$ \\ ${ }^{1}$ (Department of Microbiology, S.R.M.S.I.M.S, Bhojipura, Bareilly, India-243001) \\ ${ }^{2}$ (Department of Orthopedics, M.M.I.M.S.R, Mullana, Ambala, Haryana, India - 133207) \\ ${ }_{3}^{3}$ (Department of Microbiology, S.H.K.M, G.M.C, Nalhar, Mewat, Haryana, India - 122107) \\ ${ }^{4}$ (Department of Microbiology, M.M.I.M.S.R, Mullana, Ambala, Haryana, India - 133207)
}

\begin{abstract}
To determine the risk factors, microbiological features, clinical features and other epidemiological characteristics of Nocardia asteroides, we evaluated 500 patients with suspected pulmonary and extra pulmonary tuberculosis seen over a period of 18 months. Samples were subjected to microscopy and culture using standard protocols. Out of 500 samples, $5(1 \%)$ were found to be positive for Nocardia asteroids on the basis of growth characteristics and biochemical reactions. All the 5 (100\%) cases were detected by McClung's Carbon free broth by paraffin baiting technique. Paraffin baiting technique had significantly higher efficacy $(p<0.0001)$ as compared to Nutrient agar, Sabouraud's dextrose agar, Lowenstein-Jensen medium, paraffin agar and Gelatin agar. Antimicrobial susceptibility testing was done by disk diffusion method in blood agar and Mueller-Hinton agar plates, as recommended by the National Committee for Clinical Laboratory Standards. Amikacin and imipenem showed the 100\% sensitivity. A diagnosis of nocardiosis may have direct benefits to the patient and physician by providing a clinical diagnosis and limiting extensive diagnostic evaluations. It may also reduce the use of empirical therapy, which could be ineffective and potentially harmful.
\end{abstract}

Keywords: Antimicrobial susceptibility testing, Nocardia asteroides, Pulmonary and extra pulmonary tuberculosis, Paraffin baiting technique

\section{Introduction}

Nocardia usually is an opportunistic pathogen, with the majority of infections occurring in patients with immunosuppressive conditions. Upto one-third of patients with nocardiosis, however, are immunocompetent [1]. Irrespective of a patient's immunologic status, the isolation of Nocardia from the respiratory tract or other body source should not be regarded as a contaminant or commensal organism. Nocardia infections in humans range from chronic skin lesions to a progressive pulmonary disease with documented haematogenous dissemination to virtually any organ in the body [2].

Pulmonary nocardiosis is an acute, subacute or chronic suppurative infection with a pronounced tendency for remissions and exacerbations. Pulmonary nocardiosis mimics pulmonary tuberculosis in both clinical symptoms and radiological characteristics. In countries like India where tuberculosis is very common, anti tuberculosis drugs are started on the basis of radiology and clinical symptoms. Extra pulmonary nocardiosis is relatively common and can occur through haematogenous dissemination or a contiguous spread of necrotizing pneumonitis into the pleura, pericardium, mediastinum, and vena cava. Haematogenously disseminated nocardiosis has led to infection in the eyes (keratitis), heart valves, liver, spleen, adrenal glands, thyroid gland, and organ tissues [3].

A diagnosis of nocardiosis may have direct benefits to the patient and physician by providing a clinical diagnosis and limiting extensive diagnostic evaluations. It may also reduce the use of empirical therapy, which could be ineffective and potentially harmful. So it was decided to detect and evaluate different techniques of isolation of Nocardia species from clinically suspected cases of pulmonary and extra pulmonary tuberculosis.

\section{Materials And Methods}

Specimen collection and identification: The prospective study was carried out in Maharishi Markandeshwar Institute of Medical Sciences and Research, Mullana, Haryana, India, a tertiary care hospital over a period of 18 months. During this period, 500 samples, out of which 400 cases of suspected pulmonary and 100 cases of extra pulmonary tuberculosis (EPTS) (Table 1), received in the department of Microbiology for investigation were subjected to the study. The extra pulmonary cases included patients of lymph node enlargement, chronic osteomyelitis, infertility, tubercular meningitis, chronic abdominal and other cases suspected of tuberculosis in extra pulmonary site. The ethical committee of the hospital approved this study. A standardized form was obtained from patients documenting socio demographic information as well as clinical findings including 
predisposing conditions for nocardiosis, associated systemic diseases, duration of symptoms, previous treatment and other personal data.

In pulmonary cases, fresh sputum samples were collected in a sterilized disposable wide mouthed screw capped plastic container of $100 \mathrm{~mL}$ capacity. As far as possible, first cough on waking up in the morning was preferred, failing which a random sample was taken. Extra pulmonary cases included pus, exudate, CSF, lymph node aspirate, endometrial aspirate/curettage, pericardial fluid and peritoneal fluid. All these specimens were collected under aseptic conditions by observing standard techniques [4]. Direct microscopy was done with help of Gram's stain (Fig. 1) and partially acid-fast or modified Kinyoun's stain (Fig. 2) [4].

Pulmonary and extra pulmonary specimens were subjected to homogenization with the help of sterile glass beads in a test tube and pastle and mortar in aseptic conditions respectively. Then all the specimens were inoculated on McClung's Carbon free broth by paraffin bait technique (Fig. 3), Nutrient agar, Sabouraud dextrose agar (SDA) (Fig. 4), Lowenstein-Jensen medium (Fig. 5), paraffin agar and gelatin agar [4-6]. Incubation was done up to 6 weeks at $37^{\circ} \mathrm{C}$. The inoculated media were examined on every alternate day. Growth appearing on paraffin bait was further cultured on the SDA.

Any growth showing morphological features of Nocardia such as dry, granular, orange to yellow colored growth was subjected to Gram's and acid fast Kinyoun's staining. After staining, each of the culture showing morphological features of Nocardia species were further subjected to various biochemical reactions. On the basis of biochemical reactions, the isolates were placed into various species [4].

Antimicrobial susceptibility testing: Susceptibilities were performed on several antimicrobial agents by disk diffusion in blood agar and Mueller-Hinton agar plates, as recommended by the National Committee for Clinical Laboratory Standards (NCCLS) [7]. Each test isolate was checked for its purity and several colonies were emulsified into $50 \mathrm{~mL}$ of Mueller-Hinton broth. The inoculated flasks were incubated at $37^{\circ} \mathrm{C}$ for $24-48 \mathrm{~h}$ on a rotary shaker. Sterile glass beads ( $5 \mathrm{~mm}$ in diameter) were added to each flask to minimize the formation of clumps of nocardial growth during cultivation. The growth thus obtained was diluted 1:10 so as to give an approximate concentration of $10^{6}-10^{8} \mathrm{CFU} / \mathrm{mL}$ in accordance with the recommendations of the NCCLS [7]. The inoculum as prepared above, was spread with a cotton swab on the surface of a petri dish containing of MuellerHinton agar (MHA). Filter paper disks containing the NCCLS recommended concentrations of the antibiotics were used. The plates were incubated at $37^{\circ} \mathrm{C}$ after which the diameter of the clear zone of inhibition including the size of the $6 \mathrm{~mm}$ disk was measured in millimeters and recorded at 24 and $36-48 \mathrm{~h}$. Reference strains of Escherichia coli ATCC 25922, Pseudomonas aeruginosa ATCC 27853, Staphylococcus aureus ATCC 25923 and $N$. asteroides ATCC 19247 were used as controls to monitor the antimicrobial disk susceptibility test [7].

Table 1; pulmonary and extra pulmonary specimens from the suspected cases of Tuberculosis $(\mathrm{n}=500)$

\begin{tabular}{|l|l|}
\hline Pulmonary Samples $(\mathrm{n}=400)$ & $\begin{array}{l}\text { Number of } \\
\text { Samples }\end{array}$ \\
\hline Sputum & 400 \\
\hline Extra pulmonary Samples $(\mathrm{n}=100)$ & \\
\hline Endometrial biopsy & 40 \\
\hline Endometrial curettage/aspirate & 35 \\
\hline Ovarian cyst aspirate & 04 \\
\hline Pus samples & 05 \\
\hline Cerebrospinal fluid & 03 \\
\hline Ascitic fluid & 03 \\
\hline Pericardial fluid & 01 \\
\hline Product of conception & 02 \\
\hline Menstrual blood & 02 \\
\hline Lymph node biopsy & 01 \\
\hline Mesenteric cyst & 01 \\
\hline Bone piece & 01 \\
\hline Gastric aspirate & 02 \\
\hline
\end{tabular}

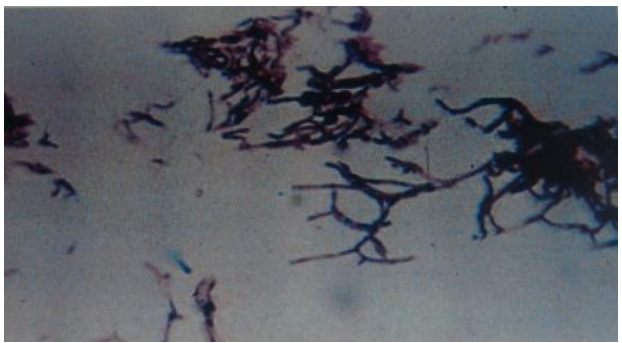

Fig. 1; Gram staining of sputum sample positive for Nocardia species 


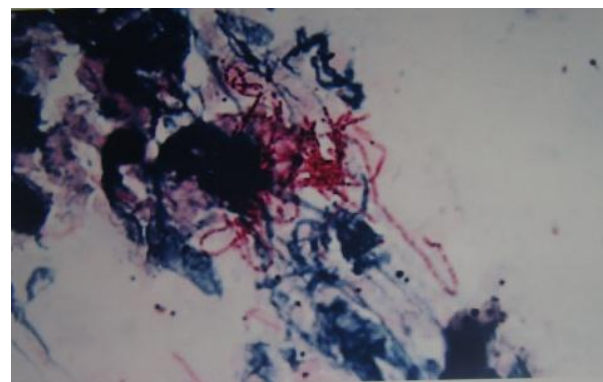

Fig. 2; Kinyoun's staining of sputum sample positive for Nocardia species

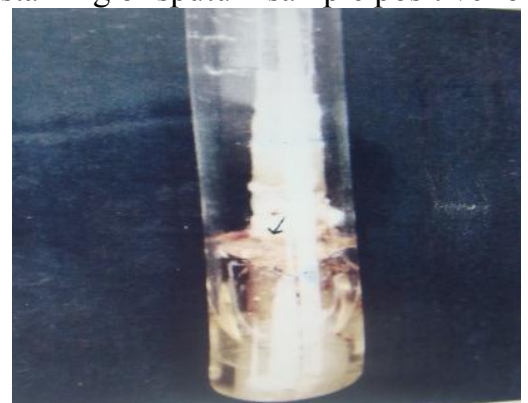

Fig. 3; Growth of Nocardia species on Paraffin Bait broth

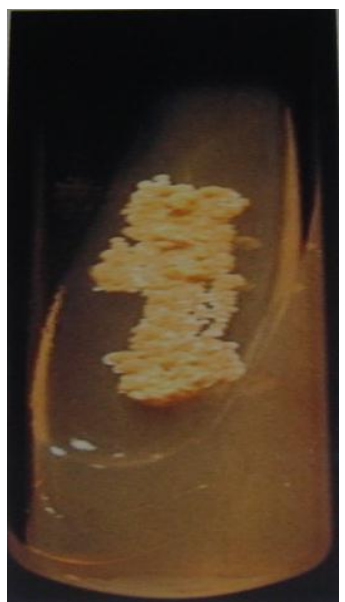

Fig. 4; Growth of Nocardia species on Sabouraud's dextrose agar

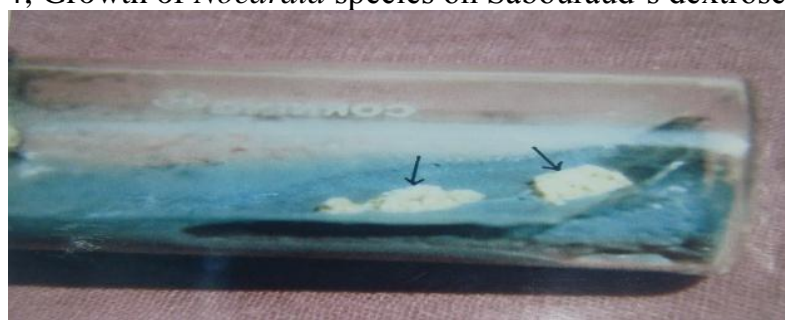

Fig. 5; Growth of Nocardia species on Lowenstein-Jensen medium

\section{Results}

Out of total 500 cases of suspected tuberculosis, a total of 400 were of pulmonary tuberculosis and remaining 100 were of extra pulmonary tuberculosis. Only one $(1 \%)$ case in the category extra pulmonary tuberculosis was found to be positive for Nocardia species. This case was derived from Endometrial biopsy. Whereas four $(1 \%)$ of pulmonary tuberculosis turned out to be nocardiosis. Thus the incidence of nocardiosis was $1 \%$ in the cases of pulmonary and as well as extra pulmonary tuberculosis.

None of the cases in high socioeconomic strata had nocardiosis. In middle socioeconomic strata the prevalence of nocardiosis was $1.08 \%$ whereas in lower strata it was $0.96 \%$. However, no statistically significant association between socioeconomic strata and nocardiosis was observed. Chronic cough and infertility were the most common clinical finding in pulmonary and extra pulmonary nocardiosis respectively.

Modified Kinyoun's stain was more sensitive as compared to Gram's stain, however, the difference 
between Gram's stain and Kinyoun's stain was significant only for Pulmonary tuberculosis and overall analysis $(\mathrm{p}<0.0001)$ (Table 2). As compared to other media, Paraffin Baiting had significantly higher efficacy $(\mathrm{p}<0.0001)$ (Table 3).

None of five Nocardia isolates decomposed tyrosine, casein and xanthine. None of five isolates fermented lactose, rhamnose and inositol. Nitrate was reduced to nitrite and urea was hydrolysed by all five isolates. Citrate was not utilized. From all the biochemical reactions, it was concluded that all the five isolates were that of Nocardia asteroides.

In antimicrobial sensitivity testing of $N$. asteroides, Amikacin, Tobramycin and imipenem showed the 100\% sensitivity while Ampicillin and Ciprofloxacin showed 80\% resistant (Table 4).

Table 2; direct microscopic examinations of samples

\begin{tabular}{|l|l|l|l|}
\hline Samples & Sample Size & Grams staining & Modified Kinyoun's staining \\
\hline Pulmonary Tuberculosis & 396 & Nil & $79(19.94 \%)$ \\
\hline $\begin{array}{l}\text { Extra pulmonary } \\
\text { Tuberculosis }\end{array}$ & 99 & Nil & $03(3 \%)$ \\
\hline Nocardiosis & 05 & $3(60 \%)$ & $02(40 \%)$ \\
\hline Total & 500 & $03(0.6 \%)$ & $84(16.8 \%)$ \\
\hline
\end{tabular}

Table 3; growth of Nocardia species on various culture media

\begin{tabular}{|l|l|l|}
\hline Media & Growth (\%) & No Growth (\%) \\
\hline Nutrient agar & $0(0 \%)$ & $5(100 \%)$ \\
\hline Lowenstein-Jenson media & $2(40 \%)$ & $3(60 \%)$ \\
\hline Sabouraud's dextrose agar & $2(40 \%)$ & $3(60 \%)$ \\
\hline Paraffin Baiting & $5(100 \%)$ & $0(0 \%)$ \\
\hline Paraffin agar & $0(0 \%)$ & $5(100 \%)$ \\
\hline Gelatin agar & $0(0 \%)$ & $5(100 \%)$ \\
\hline
\end{tabular}

Table 4; in vitro antimicrobial susceptibility testing of $N$. asteroids $(\mathrm{n}=5)$

\begin{tabular}{|l|l|l|}
\hline Antimicrobial agents & Susceptible $(\%)$ & Resistant $(\%)$ \\
\hline Ampicillin $(10 \mu \mathrm{g})$ & $1(20 \%)$ & $4(80 \%)$ \\
\hline Ciprofloxacin $(15 \mu \mathrm{g})$ & $1(20 \%)$ & $4(80 \%)$ \\
\hline Ceftriaxone $(30 \mu \mathrm{g})$ & $4(80 \%)$ & $1(20 \%)$ \\
\hline Cefotaxime $(30 \mu \mathrm{g})$ & $4(80 \%)$ & $1(20 \%)$ \\
\hline Imipenem $(10 \mu \mathrm{g})$ & $5(100 \%)$ & - \\
\hline Gentamicin $(10 \mu \mathrm{g})$ & $3(60 \%)$ & $2(40 \%)$ \\
\hline Amikacin $(30 \mu \mathrm{g})$ & $5(100 \%)$ & - \\
\hline $\begin{array}{l}\text { Trimethoprim/Sulfamethoxazole }(1.25 \quad+ \\
23.75 \mu \mathrm{g})\end{array}$ & $2(40 \%)$ & $3(60 \%)$ \\
\hline Tobramycin $(10 \mu \mathrm{g})$ & $5(100 \%)$ & - \\
\hline Amoxycillin / Clavulanic acid $(20+10 \mu \mathrm{g})$ & $3(60 \%)$ & $2(40 \%)$ \\
\hline
\end{tabular}

\section{Discussion}

Nocardiosis once considered to be a rare disease is being increasingly reported in recent years. An estimated 500-1000 new cases of nocardial infections occur every year in United States [1]. However, in India the reports are scanty possibly as a result of under reporting and lack of awareness [8]. Except for few large series, the literature mainly comprises of case reports $[9,10]$. The incidence of pulmonary disease caused by $N$. asteroides in this series was $1 \%$. These findings are similar to a study from New Delhi, where N. asteroides accounted for 1.7\% [11]. Another study from Rohtak in North India reported that Nocardia constituted 3 of 200 sputum samples [12]. Gupta et al., (1991) from Shimla reported that Nocardia constituted 2.9\% from patients of pulmonary tuberculosis [13].

In the present study, $N$. asteroides was isolated from five cases. From these five cases, three showed the Gram positive branching filaments and rest were negative on direct examination of stained smears. The reason for negativity may be that only one sample was collected from each patient and that very sample might contain less number of bacterial filaments. Panjalakshmi et al., (1980) studied 350 specimens and only two specimens revealed the presence of Gram positive, partially acid-fast filaments of bacterial width on direct examination [14]. On direct microscopic examination of stained smear, it was found that 3 smears stained with Gram's stain were positive for Gram positive branching thin filamentous organism while eighty sputum smears and four extra pulmonary samples stained with modified Kinyoun's staining technique were positive for acid fast bacilli. Out of these 84 smear positive for acid fast bacilli, eighty turned out to be Mycobacterium tuberculosis complex because they were not decolorized by $20 \%$ sulphuric acid. Of the 5 culture positive Nocardia, 3 (60\%) were screened correctly by Gram staining as gram positive, beaded, coccoid and thin branching filaments and $2(40 \%)$ by Kinyoun's method of acid-fast staining as thin, pink branching filaments in a blue background (Table 2). 
In this study, numbers of culture media were chosen for isolation of Nocardia species. These include Nutrient agar, Sabouraud's dextrose agar, Lowenstein-Jenson's media, Paraffin baiting, Paraffin agar and Gelatin agar. On conventional media (SDA), two out of five isolates could be obtained while by Paraffin baiting technique, all five isolates of Nocardia species were obtained. However, no growth was obtained on Paraffin agar and Gelatin agar. Singh et al., (1987) and Mishra et al., (1973) also reported that isolation rate of Nocardia species was twice more by Paraffin baiting technique than by conventional culture media [5,15]. Shawar et al., (1990) utilized Paraffin agar and Gelatin agar in their study [6]. They reported that Paraffin agar was good selective medium for isolation of Nocardia species. However, no growth could be obtained on the same in this study. The reason for negativity may be that only one clinical sample was collected from each patient and on the other hand Shawar et al., (1990) used sputum samples seeded with standard strain of N. asteroids [6]. Gelatin agar is selective media for the growth of $N$. brasiliensis that was not isolated in this study.

In this study nocardiosis was found in the age group of 24 to 75 years with a mean age of 49.5 years. The incidence is almost same as reported by the various authors $[14,16,17]$. The infection is common in middle age. It may be because resistance starts decreasing in this age and this age group is more susceptible to nocardiosis.

In the present study 100 suspected cases of EPTS were included. Only one (1\%) was positive for Nocardia species. The existence of Nocardia species has been reported by Beaman and Beaman (1994) [3]. A bigger study may reveal more incidences of extra pulmonary nocardiosis.

In this study, out of 500 cases, $290(58 \%)$ cases were males and $210(42 \%)$ cases were females. Out of five cases of nocardiosis male and females were three and two respectively. This incidence is similar to the incidence reported by various authors [1,17]. It seems that males were more prone to get nocardiosis. It is so because most workers and in this study large number of male patients as compared to female patients were taken. Webster (1962) who took more number of female patients as compared to males in their study, showed the more positivity in females than males (3:2) [16]. So it is concluded that sex has no relation with incidence of nocardiosis. It can occur with same frequency in both sexes.

In the present study, $N$. asteroides was the only species isolated. Some other workers also could not isolate species other than $N$. asteroides [5,13-15]. But a number of workers isolated other species including $N$. brasiliensis, $N$. farcinica and $N$. pneumonia $[12,18]$. These species may be contaminants rather than etiological agents of bronchopulmonary disease.

It is important to consider Nocardiosis in the differential diagnosis of pulmonary diseases which do not respond to antituberculous drugs and in which the sputum is negative for acid fast bacilli [19]. The pulmonary lesions due to Nocardia asteroides and Mycobacterium tuberculosis closely mimic classical tuberculosis clinically and radiologically. In countries like India where tuberculosis is very common, treatment of tuberculosis is started on basis of radiology and clinical symptoms. A classic radiographic picture of tuberculosis that is unresponsive to medication should raise the suspicion of Nocardia infection [9]. Although, nocardiosis resembles tuberculosis, the first line anti tubercular drugs has no role to play in its treatment [20]. Early diagnosis can help to effectively manage pulmonary and extra pulmonary nocardiosis, but requires a high index of clinical suspicion and microbiology laboratory support. A high index of clinical suspicion of this infection should exist in patients with a rural background and presence of typical clinical pictures. Detection of thin, delicate, intertwined, narrow, branching filaments, which are gram positive, often appearing as beaded or coccoid forms and are also $1 \%$ acid-fast staining positive, should prompt the treating physician to initiate the appropriate therapy for Nocardia infection.

Clinical outcome: The treatment of choice for this infection includes sulphonamides and, more recently, Trimethoprim/sulfamethoxazole (TMP-SMX), associated with surgical drainage wherever required [21]. However, failure with TMP-SMX has been reported. Interpretation of antimicrobial testing of Nocardia species remains a problem [22]. Inoculum preparation, incubation of isolates and reading of inhibitory end-points may be difficult because of the characteristics of slower growth and tendency for elongation and branching. The NCCLS has recently organized a working group to standardize methods for susceptibility testing in the aerobic actinomycetes, including Nocardia species. The results of sensitivity testing do not always correlate with clinical outcome [23], so that this fatal evolution, together with the appearance of TMP-SMX-resistant $N$. asteroides, provide a rational basis for considering combined therapy and alternative agents with a proven clinical response. It was observed that among these agents, netilmicin, amikacin and imipenem showed both in vitro susceptibility and clinical resolution [24]. Evidence from experimental studies using antimicrobial combinations has demonstrated synergy in vitro, which has also been supported by clinical observation with imipenem-amikacin, imipenem-cefotaxime and amikacin-cefotaxime $[25,26]$.

Resolution of the disease was defined as eradication of Nocardia species, together with clinical and radiological improvement. Nocardia was isolated from five cases. All these five cases were diagnosed clinically as tuberculosis and were on anti tubercular treatment. The clinical end-points were freedom from fever, which 
was achieved during the first few days (range 2-5 days) after treatment, with reduction in purulence of sputum. Radiological improvement lagged behind clinical parameters, with complete resolution in 1-3 months and partial resolution in one patient. Pulmonary and extra pulmonary nocardiosis resolved in all five patients. The duration of therapy is variable, and depends on the localization of the lesions and the immune status. Antibiotic therapy was started empirically, with subsequent modification according to antibiotic sensitivity tests and clinical evolution. Patients were treated with intravenous imipenem followed by oral Trimethoprim/sulfamethoxazole, with resolution at 2 months.

\section{Conclusion}

Nocardiosis is more commonly associated with suspected cases of pulmonary tuberculosis as compared to suspected cases of extra pulmonary tuberculosis. Modified Kinyoun's staining is more sensitive method for screening Nocardia in suspected cases of nocardiosis. Paraffin baiting technique is more reliable than routine culture media for $N$. asteroides. It has been observed that media composition and inoculum size considerably influence the in vitro results. Each test technique requires a uniform homogeneous suspension but this may not be achievable with all strains, since nocardiae grow as long branching filaments that fragment and clump. In vitro antimicrobial susceptibility testing for Nocardia species needs standardization, both in terms of methodology and correlation with in vivo therapeutic response. The clinical outcome of therapy depends on the site and extent of diseases and underlying host factors. Most of the patients have favourable outcomes and showed good response to treatment, in almost all cases.

[1] B.L. Beaman, J. Burnside, B. Edwards, and W. Causey, Nocardial infections in the United States, 1972-1974, J. Infect. Dis., 134(3), 1976, 286-289.

[2] W.A. Curry, Human Nocardiosis: a clinical review with selected case reports, Arch. intern., 140, $1980,818-826$.

[3] B.L. Beaman, and L. Beaman, Nocardia species: host-parasite relationships, Clin. Microbiol. Rev. 7(2), 1994, $213-264$

[4] M. Goodfellow, Actinomycetes: Actinomyces, Actinomadura, Nocardia, Streptomyces and related genera, In J.G. Collee, B.P. Marmion, A.G. Fraser, A. Simmons (Ed.), Mackie \& McCartney, Practical Medical Microbiology, (London: Churchill Livingstone, 1996) 343-359.

[5] M. Singh, R.S. Sandhu, and H.S. Randhawa, Comparison of Paraffin baiting and conventional culture techniques for isolation of Nocardia asteroids from sputum, J. Clin. Microbiol., 25, 1987, 176-177.

[6] R.M. Shawar, D.G. Moore, and M.T. Larocco, Cultivation of Nocardia species on chemically defined media for selective recovery of isolates from clinical specimens, J. Clin. Microbiol., 28, 1990, 508-512.

[7] National Committee for Clinical Laboratory Standards (NCCLS). Performance standards for antimicrobial disk susceptibilty tests. Approved standard M2-A5. Villanova, PA: National Committee for Clinical Laboratory Standards, 1994.

[8] M.R. Shivaprakash, P. Rao, J. Mandal, M. Biswal, S. Gupta, P. Ray, and A. Chakrabati, Nocardiosis in a tertiary care hospital in north India and review of patients reported from India, Mycopathologia, 5, 2007, 267-274.

[9] V. Chopra, G.C. Ahir, G. Chand, and P.K. Jain, Pulmonary nocardiosis mimicking pulmonary tuberculosis, Indian J. Tuberculosis, 48, 2001, 211-213.

[10] R. Chaudhary, A. Aher, V. Rastogi, and T.K. Prabhu, Case of mixed pulmonary infection by Nocardia and Mycobacterium tuberculosis, I.J.P.M., 52, 2009, 295.

[11] H.S. Randhawa, and Z.U. Khan, Pulmonary and systemic nocardiosis, Indian J. Chest Ds. Allied Sciences, $29,1977,32-44$.

[12] A.K. Malik, U. Sabharwal, and T.D. Chug, Pulmonary Nocardiosis, Indian J. Pathol. Microbiol., 23, 1980, $209-211$.

[13] M.L. Gupta, P.S. Grover, B.K. Bhardwaj, Nocardia associated with pulmonary tuberculosis, J. Comm. Dis. 23, 1991, 157-159.

[14] V.V. Pankajalakshmi, V.V. Taralakshmi, S. Subramaniam, and S. Arumugam, Nocardia species from bronchopulmonary infections and mycetomas, Sabouraudia, 18, 1980, 11-18.

[15] S.K. Mishra, H.S. Randhawa, and R.S. Sandhu, (1973) Observations on Paraffin baiting as a laboratory diagnostic procedure in nocardiosis, Mycopathologia, 51, 1973, 147-157.

[16] B.H. Webster, Bronchopulmonary nocardiosis, Am. J. Med. Sciences, 224, 1962, 40-43.

[17] D.L. Palmer, R.L. Harvey, and J.K. Wheeler, Diagnostic and therapeutic considerations in Nocardia asteroides infection, Journal of Medicine, 53, 1974, 391-401.

[18] Z.H. Helal, M.I. Khan, A.M. Sief El-Din, and S.A. Eissa, Detection and characterization of Nocardia from patients diagnosed as Tuberculosis in Egypt, International J. Biomed. Science, 4, 2008, 179-184.

[19] M. Dias, B. Antony, and H. Pinto, Spectrum of nocardiosis - A report of three cases, J. Clin. Diagno. Res. 3, 2009 , $1682-1684$.

[20] A. Kumar, A. Mehta, G. Kavathia, and M. Madan, (2011) Pulmonary and Extrapulmonary Tuberculosis along with Pulmonary Nocardiosis in a patient with human Immunodeficiency Virus Infection: A case report, J. Clin. Diagno. Res., 5, 2011, $109-111$.

[21] P.I. Lerner, (1985) Nocardia species. In G.L. Mandell, R.G. Douglas, J.E. Bennett (Ed.), Principles and Practice of Infectious Diseases, (New York: Wiley, 1985) 192-206.

[22] M.A. Saubolle, In vitro susceptibility testing of clinical isolates of Nocardia, Clin. Microbiol. News, 15, 1993, 169-172.

[23] E.H. Runyon, Nocardia asteroides: studies of its pathogenicity and drug sensitivities, J. Lab. Clin. Med., 37, 1951, 713-725.

[24] C. Farina, P. Boiron, A. Goglio, and F. Provost, Human nocardiosis in northern Italy from 1982 to 1992 : Northern Italy Collaborative Group on Nocardiosis, Scan. J. Infect. Dis., 27, 1995, $23-27$.

[25] M.E. Gombert, and T.M. Aulicino, Synergism of imipenem and amikacin in combination with other antibiotics against Nocardia asteroides, Antimicrob. Agents. Chemother., 24, 1983, 810-811.

[26] M.E. Gombert, T.M. Aulicino, L. du Bouchet, G.E. Silverman, and W.M. Sheinbaum, Therapy of experimental cerebral nocardiosis with imipenem, amikacin, trimethoprimsulfamethoxazole, and minocycline. Antimicrob. Agents. Chemother. 30, 1986, $270-273$. 